\title{
Relaxational processes in the one-dimensional Ising model with long-range interactions
}

\author{
Yusuke Tomita \\ College of Engineering, Shibaura Institute of Technology, Minuma-ku, Saitama 337-8570, Japan
}

(Dated: June 20, 2018)

\begin{abstract}
Relaxational processes in ordered phases of one-dimensional Ising models with long-range interactions are investigated by Monte Carlo simulations. Three types of spin model, the pure ferromagnetic, the diluted ferromagnetic, and the spin glass models, are examined. The effective dimension of the one-dimensional systems are controlled by a parameter $\sigma$, which tunes the rate of interaction decay. Systematical investigations of droplet dynamics, from the lower to the upper critical dimension, are conducted by changing the value of $\sigma$. Comparing numerical data with the droplet theory, it is found that the surface dimension of droplets is distributed around the effective dimension. The distribution in the surface dimension makes the droplet dynamics complex and extremely enhances dynamical crossover.
\end{abstract}

\section{INTRODUCTION}

Probing dynamical properties is an indispensable mean to investigate magnetic and/or dielectric materials. To extract the dynamical properties, several methods (e.g., x-ray scattering, neutron scattering, nuclear magnetic resonance, electron spin resonance, muon spin resonance, etc.) are adopted in various research fields. While a couple of one-shot experiments could extract characters of a material, a large number of experiments on a group of similar substances will be required to acquire a fundamental understanding of an intriguing phenomenon. In order to obtain an organized view on the phenomenon, an effective framework which visualizes relations between corresponding experiments is important. The Cole-Cole plot [1] is an example of such framework. It is a plot of the real part and the imaginary part of the permittivity which visualizes dielectric relaxation of a substance and makes it easier to classify dielectric materials by comparing each Cole-Cole plot. Another example is the Angell plot for glassforming liquids 2]. It plots a viscosity of a substance as a function of scaled temperature, and visualizes whether it is fragile or strong liquid. These frameworks categorize dynamics of various substances in a simple manner, and we need a theory that integrates the findings of the dynamics in many-body systems into a unified perspective. Though dynamics at criticalities are well studied [3, 4], dynamics in ordered phases are not clarified sufficiently.

The droplet theory is one of theories dealing with dynamics in ordered phases of many-body systems. The droplet theory has succeeded in explaining dynamics of pure ferromagnetic models 5, 6], ferromagnetic models with randomness [5], and the spin glass model [7, 8]. The droplet model assumes a shape of the free-energy landscape of droplets, which are domains of an ordered state, and deduces a relaxational behavior of the system. Dynamical properties relate closely to the shape of droplets, which depends on both the dimensionality of the system and the type of the interactions. To develop understanding of ordered states, a comprehensive study on relations between dynamical properties and the shape of droplets is required. The one-dimensional (1D) Ising model with long-range interactions [Eq. (10] provides a suitable test ground for the comprehensive study. Though the spin model looks simple, it exhibits pure ferromagnetic, ferromagnetic with randomness, and spin glass phases by changing the distribution of interactions and tuning the rate of interaction decay. Tunable long-range interactions of the model enables to investigate the relaxational processes in a continuous manner from the lower to the upper critical dimension. Through numerical analyses of the $1 \mathrm{D}$ Ising model, we examine the droplet theory as a suitable framework to describe the mechanism of various dynamics in many-body spin systems.

This paper is organized as follows: In Sec. II, we briefly review preceding studies on the $1 \mathrm{D}$ Ising model with longrange interactions and the droplet theory. Details on numerical calculations are given in Sec. III. Section IV presents results obtained by Monte Carlo simulations of the $1 \mathrm{D}$ Ising model. Section $\mathrm{V}$ is devoted to the discussion. The summary of this paper is presented in Sec. VI. A detail explanation of $O(N)$ Monte Carlo method [9 11], which is a key algorithm to achieve numerical calculations of systems with long-range interactions with reasonable computational cost, is given in Appendix

\section{REVIEWS OF THE 1D ISING MODEL WITH LONG-RANGE INTERACTIONS AND THE DROPLET THEORY}

This section presents brief reviews on the 1D Ising model with long-range interactions and the droplet theory.

The Hamiltonian of the 1D Ising model is given by

$$
\mathcal{H}=-\sum_{i<j} \frac{J_{i j}}{r_{i j}^{\sigma}} S_{i} S_{j}
$$

Here, $S_{i}(\in\{1,-1\})$ represents the Ising spin variable at site $i, J_{i j}$ is the exchange interaction between $i$ and $j, r_{i j}$ is the distance between $i$ and $j$, and $\sigma$ is the tuning parameter of long-range interaction. Despite its dimensionality and simple appearance, the model possesses various features which are controlled by the model parameters: 
the complexity of the interactions, $J_{i j}$, and the tuning parameter of the interaction decay, $\sigma$.

The section consists of three subsections. In the first subsection, we review preceding studies on pure ferromagnetic models. The second subsection gives reviews on diluted ferromagnetic models. Spin glass models are reviewed in the third subsection.

\section{A. Pure ferromagnetic model}

The pure ferromagnetic model is characterized by a uniform ferromagnetic interaction:

$$
J_{i j}=J(>0) \quad(\text { for any } i \text { and } j) .
$$

The interaction decay with increasing distance between spin pairs is tuned by the parameter $\sigma$ : The long-range interaction is irrelevant when $\sigma>2$, and the system does not exhibit the ferromagnetic order at any finite temperature. The critical phenomena of the system belong to the universality class of the mean-field model, when $\sigma \leq 3 / 2$. At $\sigma=2$, the Kosterlitz-Thouless (KT) transition [12 14] appears at a finite temperature. In the range $3 / 2<\sigma<2$, the universality class of the ferromagnetic phase transition depends on the value of $\sigma$. Therefore, by varying $\sigma$ from 2 to $3 / 2$, we can continuously survey the Ising model from the lower critical $[d(\sigma=2)=1]$ to the upper critical $[d(\sigma=3 / 2)=4]$ dimension. It should be noticed that the 1D Ising model on the trace of the effective dimension $[d(\sigma)]$ does not correspond to the nearestneighbor model. For example, the 2D nearest-neighbor model exhibits a logarithmic divergence of the specific heat while the critical exponent of the specific heat of the $1 \mathrm{D}$ model is positive in the range of $3 / 2<\sigma<2$.

While we have little analytical results on dynamics of the Ising model, rigorous results of dynamics in the paramagnetic phase are given by Glauber [15]. Using his results, the time-delayed correlation function $C(k ; t)$ is given by

$$
\begin{aligned}
C(k ; t) & =\left\langle\tilde{S}_{-k}(0) \tilde{S}_{k}(t)\right\rangle \\
& =\xi(k) \exp [-t / \tau(k)],
\end{aligned}
$$

where $\tilde{S}_{k}(t)$ is the Fourier transform of the Ising spin at time $t, \xi(k)$ is the correlation length for the wave number $k, \tau(k)$ is the lifetime for the wave number $k$, and $T$ is the temperature (the Boltzmann constant $k_{\mathrm{B}}$ is set to unity). The angle brackets $\langle\cdots\rangle$ denote a thermal average. The Fourier transform of the Ising spin $\tilde{S}_{k}(t)$, the correlation length $\xi(k)$, and the lifetime $\tau(k)$ are, respectively, given by

$$
\begin{aligned}
\tilde{S}_{k}(t) & =\frac{1}{L} \sum_{r} S_{r}(t) e^{i k r}, \\
\xi(k) & =[\cosh (2 J / T)(1-\gamma \cos k)]^{-1}, \\
\tau(k) & =[a(1-\gamma \cos k)]^{-1},
\end{aligned}
$$

where $L$ is the number of spins, $\gamma=\tanh 2 J / T$, and $a$ is a nonuniversal constant. The result shows that the correlation function in the paramagnetic phase decays exponentially with time, and the lifetime is proportional to the correlation length.

The correlation function of the Ising model in the mean-field region is given by several authors [16 18]. The time-delayed correlation function $C(t)$ in the ordered phase is

$$
\begin{aligned}
C(t) & =\langle S(0) S(t)\rangle \\
& \sim \frac{C_{0}^{2}}{\left(C_{0} / C_{\infty}\right)^{2}-\left[\left(C_{0} / C_{\infty}\right)^{2}-1\right] e^{-t / \tau}},
\end{aligned}
$$

where $C_{0}$ and $C_{\infty}$ are nonuniversal constants. The result shows that the correlation function in the ordered phase shows an exponential convergence as well as in the paramagnetic phase.

In the intermediate dimension $(1<d<4)$, there is no rigorous result of dynamics in the ordered phase, but deduced forms of the autocorrelation function by the droplet theory are available. When the spatial dimensionality $d$ is sufficiently low $(d<3)$ in the nearestneighbor interaction model, Huse and Fisher have shown that the autocorrelation function $C_{i}(t)$ at site $i$ shows the Kohlrausch-Williams-Watts stretched exponential decay as [5],

$$
\begin{aligned}
C_{i}(t) & =\left\langle S_{i}(0) S_{i}(t)\right\rangle-\left\langle S_{i}\right\rangle^{2} \\
& \sim \exp \left[-(t / \tau)^{(d-1) / 2}\right] .
\end{aligned}
$$

This stretched exponential decay comes from the emergence of large-scale droplets. Since a lifetime of largescale droplet is long, the excited droplets dominate the dynamics of the system. On the other hand, such largescale droplets do not emerge at a high dimensionality. Larger droplets are much more affected by thermal fluctuations since the surface area of droplets increases proportionally with the linear size $l$ to the power of $d-1$, $l^{d-1}$. For $d>3$, fluctuations of average size droplets dominate the correlations, and the system shows a simple exponential decay, $C_{i}(t) \sim \exp [-(t / \tau)]$. The prediction of the droplet theory indicates that the 1D Ising model will show a simple exponential decay in high enough dimension. In other words, there is a critical value of $\sigma_{c}$ $\left[d\left(\sigma_{c}\right)=3\right]$ where the form of the autocorrelation function changes.

\section{B. Diluted ferromagnetic model}

The diluted ferromagnetic model possesses randomness without frustration, and it serves a suitable test ground to study effects of randomness. There are two types of dilution, site- and bond-dilution. In the present paper, we deal with a bond-dilution model whose Hamiltonian is given by Eq. (10) with randomly diluted interactions,

$$
J_{i j}= \begin{cases}J(>0) & (\text { with probability } 1 / 2) \\ J^{\prime}(=0) & (\text { with probability } 1 / 2)\end{cases}
$$


The value of the diluted interaction is chosen so as to maximize effects of randomness. The disconnections of the interaction $\left(J^{\prime}=0\right)$ bring a lowering of a transition temperature, and the ordered state in a low temperature is strongly affected by the geometry of the interaction network. The geometrical effect is stronger at larger $\sigma$ since the phase transition temperature decreases as increases $\sigma$. The details of the effect is discussed in Sec. $\nabla$

The phase diagram of the diluted model $\left(J^{\prime} \geq 0\right)$ is almost the same as the pure model. However, according to the Harris criterion [19], the universality class of the diluted model in the range of $3 / 2<\sigma<2$, where the critical exponent of the specific heat of the pure model $\alpha$ is positive, is altered by the dilution. On the other hand, the critical exponent $\alpha$ is zero when $\sigma=2$ and $\sigma \leq 3 / 2$, and the dilution will be irrelevant to the universality class. For systems that exhibit the KT transition, it will not be a trivial question whether the dilution alters the universality class or not, but it seems irrelevant regarding numerical studies of the two-dimensional diluted XY model [20, 21].

By assuming the dilution being not so strong, Huse and Fisher derived that the autocorrelation function shows a power-law decay,

$$
\overline{C_{i}}(t) \sim t^{-x(T)},
$$

where the overbar denotes the sample average of the interaction realization. The exponent $x(T)$ will depend on the temperature and nonuniversal details of the system [5]. The arrangement of diluted bonds could strongly affect the relaxation at low temperatures $\left(T \ll T_{c}\right)$ since the exponent $x(T)$ depends on the rate of the creation and the annihilation of large droplets. However, thermal fluctuations blur the details of the arrangement of diluted bonds, and $x(T)$ presumably approaches a universal value near the transition temperature.

\section{Spin glass model}

Interactions of the spin glass model consist of ferromagnetic and antiferromagnetic bonds, and the random arrangement of bonds brings about frustration in the system. In the present paper, we deal with a random bond model whose Hamiltonian is given by Eq. (11) with randomly mixed interactions,

$$
J_{i j}= \begin{cases}J & (\text { with probability } 1 / 2), \\ -J & (\text { with probability } 1 / 2) .\end{cases}
$$

Though the bimodal distribution in the exchange interaction does not bring about frustration in the 1D model when nearest-neighbor interactions are dominance, frustration emerges when interactions are sufficiently longranged. Kotliar, Anderson, and Stein showed that the spin glass phase appears when $\sigma<1$ : the universality class of the model belongs to that of the mean-field for $\sigma<2 / 3$, and that depends on $\sigma$ for $2 / 3<\sigma<1[22$.
As in the diluted model, the relaxation of the system is altered further by frustrated interactions. Fisher and Huse derived the logarithmic decay of autocorrelation function,

$$
\bar{C}(t) \sim\left[\ln \left(t / \tau_{0}\right)\right]^{-\phi},
$$

where $\tau_{0}$ and $\phi$ are, respectively, a microscopic time scale and a nontrivial exponent [7, [8]. The logarithmic decay originates from the distributions of the droplet free energy $F_{L}$ and the droplet barrier for annihilation of droplets $B_{L}$. Both of the two distributions have broad distribution and different size dependencies, $F_{L} \sim L^{\theta}$ and $B_{L} \sim L^{\psi}$. The exponent $\phi$ in Eq. (13) is the ratio of the exponents, $\phi=\theta / \psi$.

In the mean-field regime, the time-delayed correlation function at site $i$ in the spin glass phase, $\bar{C}_{i}(t)$, is given by [23]

$$
\bar{C}_{i}(t)=\frac{1-C_{0}}{(1+a t)^{1 / 2}}+C_{0},
$$

where $a$ and $C_{0}$ are constants. Unlike the case of the ferromagnetic model, the correlation function shows the power-law decay with an exponent $1 / 2$.

\section{METHOD}

While the 1D Ising model with long-range interactions is a suitable spin model for analytical studies, the large computational cost of scanning all the interactions proportional to the square of the system size, $O\left(L^{2}\right)$, hampers numerical studies. To overcome the numerical difficulty in systems with long-range interactions, Fukui and Todo proposed $O(N)$ cluster Monte Carlo (MC) method 9]. The $O(N)$ cluster MC method was successfully applied to study the single-spin-flip dynamics in 1D Ising models with power law decaying interactions and nontrivially frustrated systems [10], and 2D Heisenberg dipolar lattices [11]. The details of the algorithm are given in Appendix.

To eliminate the edges of the system, the periodic boundary condition is imposed to all simulations. For the pure and the diluted ferromagnetic models, all of the contributions from supercells are summed up. When $\sigma>1$, the summation is easily executed as

$$
\begin{aligned}
\frac{1}{\tilde{r}_{i j}^{\sigma}} & =\sum_{n=-\infty}^{\infty} \frac{1}{\left(r_{i j}+n L\right)^{\sigma}} \\
& =\frac{1}{L^{\sigma}}\left[\zeta\left(\sigma, r_{i j} / L\right)+\zeta\left(\sigma,\left(L-r_{i j}\right) / L\right)\right],
\end{aligned}
$$

where $\tilde{r}_{i j}$ and $\zeta(\sigma, r)$ are, respectively, the effective distance between site $i$ and $j$ and the Hurwitz zeta function,

$$
\zeta(\sigma, r)=\sum_{n=0}^{\infty} \frac{1}{(n+r)^{\sigma}} .
$$


However, the summation does not converge when $\sigma \leq 1$. In order to implement the periodic boundary condition when $\sigma \leq 1$, the chord distance [24] is implemented in the spin glass model: We place a spin $S_{i}$ equidistantly on a ring of length $L$, and the distance $r_{i j}$ is described by

$$
r_{i j}=\frac{L}{\pi} \sin \left(\frac{\pi|i-j|}{L}\right) .
$$

The slow decay of the interaction causes a large transition temperature. Therefore, we rescale the interaction $J$ to $c(\sigma, L) J$ as

$$
c(\sigma, L)=\sqrt{\frac{L}{2 J \sum_{i<j} r_{i j}^{-2 \sigma}}} .
$$

This rescaling adjusts the transition temperature at $\sigma=$ 0 to unity and makes the transition temperature to be moderate for $\sigma>0$. In the $\mathrm{MC}$ simulation, the interactions considered above are employed.

To investigate the dynamical properties, we calculate time-delayed correlation functions. In the pure and the diluted ferromagnetic models, the correlation function is calculated by Eq. (3). The time-delayed correlation function in the spin glass model is defined as

$$
\bar{C}(k ; t)=\overline{\left\langle\tilde{q}_{-k}(0) \tilde{q}_{k}(t)\right\rangle},
$$

where $\tilde{q}_{k}(t)$ is the Fourier transform of the EdwardsAnderson order parameter 25] at time $t$, which is given by

$$
\tilde{q}_{k}(t)=\frac{1}{L} \sum_{r} S_{r}^{(1)}(t) S_{r}^{(2)}(t) e^{i k r}
$$

Here, the upper suffixes (1) and (2) denote the replica indexes. The time-delayed correlation function [Eq. (19)] is different from the autocorrelation function considered in the droplet theory [5, 7, 8]. Whereas the droplet theory deals with the time-delayed correlation of a local spin at site $i$, Eq. (19) deals with that of the macroscopic order parameter.

\section{RESULTS}

In this section, results obtained by Monte Carlo simulations on three different models, pure ferromagnetic, dilute ferromagnetic, and spin glass, are shown. In what follows, we use $J$ as a unit of temperature. Error bars of obtained data are omitted since large error bars impair the visibility of figures. Large statistical errors of the data mainly come from small values of observables. Though statistical errors at each point are large, trends of decay in autocorrelations are clearly observed as we see below.

\section{A. Pure ferromagnetic model}

This subsection gives results obtained by Monte Carlo simulations of the 1D pure ferromagnetic model. Monte Carlo simulations are executed for several values of $\sigma$, a parameter of long-range interactions. The system size is set as $L=2^{20}\left(\simeq 1.0 \times 10^{6}\right)$. The system is equilibrated by combination use of annealing and a cluster flip update [9]. $10^{7}$ Monte Carlo steps are executed for measurement of autocorrelation functions, and 10 independent samples are simulated for obtaining good statistics.

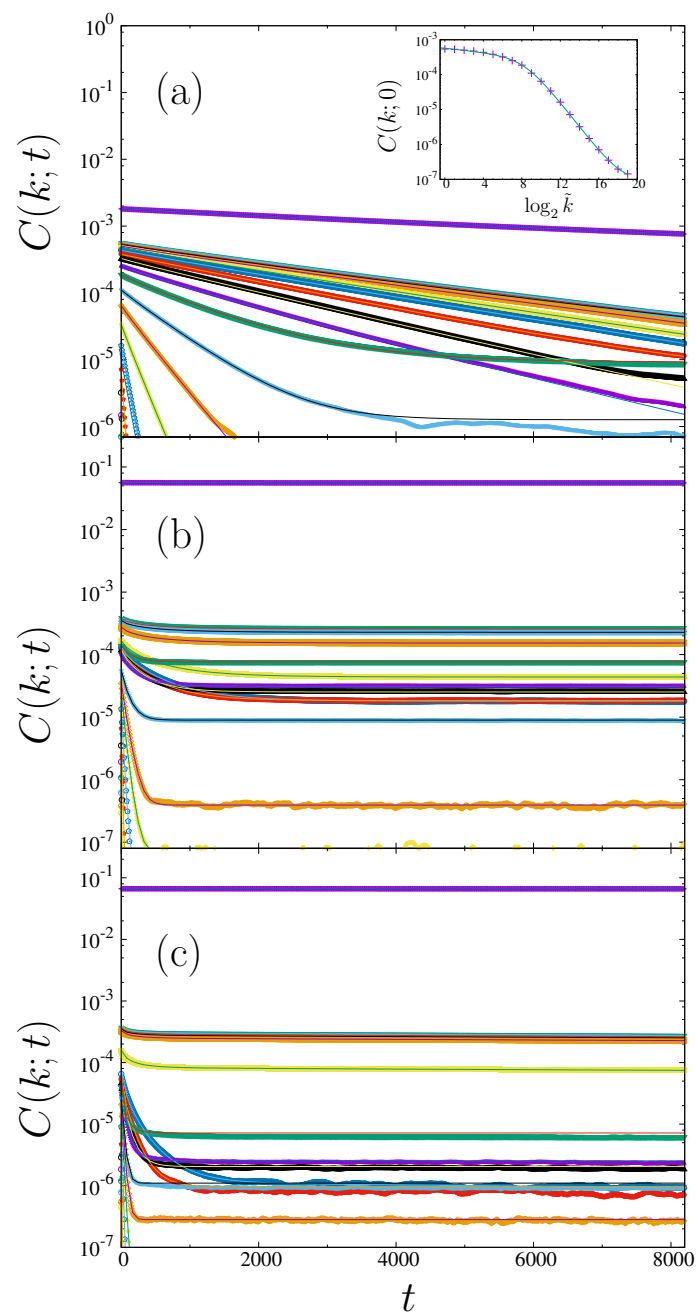

FIG. 1. (Color online) Autocorrelation functions of the pure ferromagnetic model at (a) $\sigma=2$, (b) $\sigma=1.8$, and (c) $\sigma=1.6$. Topmost lines in each figure are autocorrelation functions of $k=0$. Following to the function of $k=0$, autocorrelation functions of $\log _{2} k=0,1,2, \ldots$ align from top to bottom in almost ascending order. Inset shows $k$-dependence of the autocorrelation functions at $\sigma=2$ and $t=0$.

As reviewed in Section II the pure ferromagnetic model shows the KT transition at a finite temperature when $\sigma=2$. Autocorrelation functions would exhibit simple exponential decays even below the transition temperature since there is no true long-range order. Unlike 
those in normal paramagnetic phase, the autocorrelation function in the KT phase at time $t, C(k ; t)$, depends on a power of a wave number $k$,

$$
C(k ; t) \propto k^{-\eta} \exp [-t / \tau(k)],
$$

where $\eta$ is the critical exponent of the correlation function and $\tau(k)$ is the lifetime for $k$. The autocorrelation functions in the KT phase $(T=1.78)$ obtained by Monte Carlo simulation are plotted in Figs. 1(a). Wave numbers in Figs. 1(a) are zero and $\tilde{k}=2^{n}(n=0,1,2, \ldots, 19)$, where $\tilde{k}$ denotes scaled wave number, $\tilde{k}=L k / 2 \pi$. Curves of the autocorrelation functions at $t=0(\mathrm{MCS}=0)$ ought to be equally-spaced in the logarithmic scale if their relaxations are described by Eq. (21). But Figs.1(a) shows distances between neighboring curves become wider as $\tilde{k}(\geq 1)$ increases. To examine the $k$-dependence of autocorrelation functions at $t=0, \mathrm{I}$ assumed a $k$-dependence,

$$
C(\tilde{k} ; 0)=C_{1} \frac{k^{-\eta}}{1+(k / \kappa)^{\phi}}+C_{2},
$$

rather than Eq. (21). Constants $C_{1}, C_{2}$, and $\kappa$ are nonuniversal constants. It is known that $\eta=0$ and $\phi=2$ when the system is in the mean field region [26]. The formula, Eq. (22), conforms to Eq. (21) at $t=0$ when $\phi=0$. The result is shown in inset of Figs. 1(a). Estimated values are $\eta=0.10, \phi=1.1$, and $\kappa=2.0 \times 10^{-3}$. The result indicates that the system seems to be in the KT phase in the range of $k<\kappa$ whereas the system seems to be in an ordered state in the range of $k>\kappa$. The emergence of the ordered state can be explained by the characteristic length of ferromagnetic clusters of Ising spin. At a low enough temperature, larger ferromagnetic clusters are more stable than smaller ones. Because of the discreteness of the Ising spin, there is little cluster whose size is smaller than $1 / \kappa$ at such a low temperature. Therefore the system seems ordered in the range of $k>\kappa$.

As mentioned in Sec. IIA the relaxation of autocorrelation function depends on the dimensionality of the droplet, which is directly affected by the parameter $\sigma$. According to the results by Tang, Nakanishi, and Langer [6], there will be a critical parameter $\sigma_{c}$ : A stretched exponential decay will be observed when $\sigma_{c}<\sigma<2$, while a relaxation will be simple exponential decay when $\sigma<\sigma_{c}$. Figures 1(b) and (c) show exponential decays observed in an ordered phase $T=2.48$ at $\sigma=1.8$ and $T=3.50$ at $\sigma=1.6$, respectively. In order to examine forms of relaxations of order parameter, multiparameter fittings are performed while assuming a fitting form,

$$
C(k ; t)=C_{0} \exp \left[-(t / \tau(k))^{\beta(k)}\right]+C_{\infty},
$$

where $\beta(k)$ is a stretching exponent for $k$ and $C_{0}$ and $C_{\infty}$ are nonuniversal constants. The fitting form Eq. (23) well fits data when $\sigma \geq 1.7$, but the form is not good enough to fit data when $\sigma \leq 1.6$. By adding another exponential term to the form, fitting results are fairly improved. The fitting form applied to systems with $\sigma \leq 1.6$ is

$$
\begin{aligned}
C(k ; t)= & C_{0} \exp \left[-(t / \tau(k))^{\beta(k)}\right] \\
& +C_{2} \exp \left[-\left(t / \tau_{2}(k)\right)^{\beta_{2}(k)}\right]+C_{\infty},
\end{aligned}
$$

where $\beta(k)$ and $\beta_{2}(k)$ are stretching exponents for $k, \tau(k)$ and $\tau_{2}(k)(>\tau(k))$ are lifetimes for $k$, and $C_{0}, C_{2}$, and $C_{\infty}$ are nonuniversal constants. The necessity of two exponential terms means that there is another non-negligible mode in relaxational processes when $\sigma \leq 1.6$. The origin of the two non-negligible modes is discussed in Sec. V

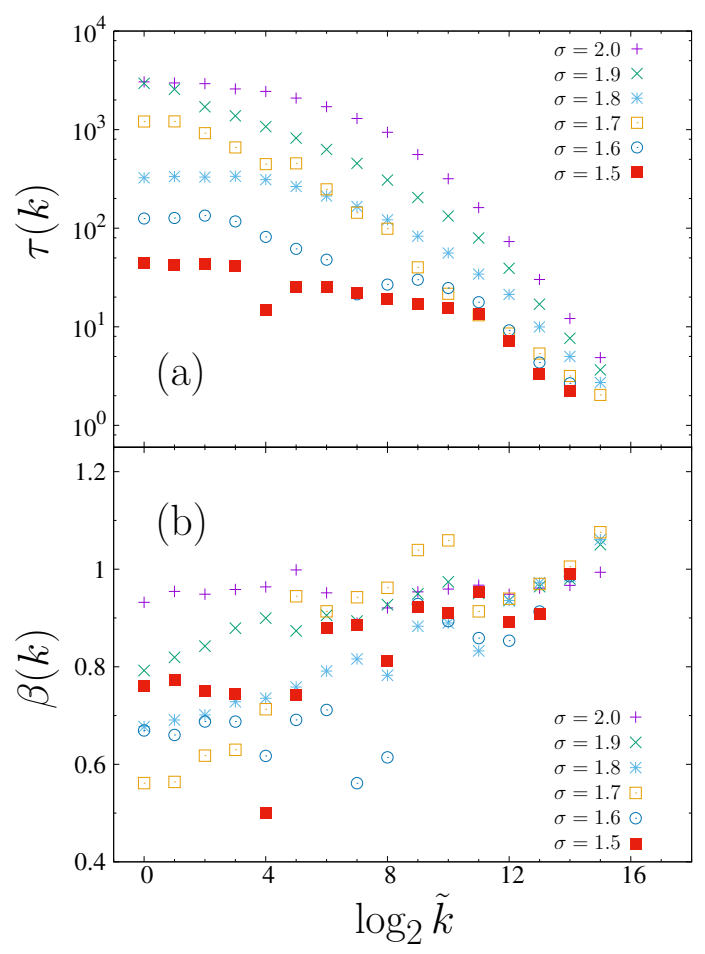

FIG. 2. (Color online) (a) Lifetimes for several $\sigma$ 's as functions of $\tilde{k}$. Lifetimes tend to decrease as $\tilde{k}$ increases; lifetimes of small droplets are shorter than those of larger ones. (b) Stretching exponents for several $\sigma$ 's as functions of $\tilde{k}$. The exponents are nearly continuous functions of $\tilde{k}$ when $\sigma \geq 1.8$, whereas they have a discontinuous jump at $\tilde{k} \sim 5$ when $\sigma \leq 1.7$.

The stretching exponents and lifetimes are plotted in Figs. 2. The exponents $\beta(k)$ at $\sigma=2.0$ are almost independent of $k$. The value of $\beta$ is unity when the long-range order is absent. Therefore values of $\beta(k)$ are expected to be unity since the KT phase does not have the true longrange order. The obtained values of $\beta(k)$ are, however, slightly smaller than unity. This would be caused by the finiteness of the system size. The correlation length is larger than the system size, so that the system seems as if it has true long-range order.

The values of $\beta(k)$ at $\sigma=1.9$ and $\sigma=1.8$ increase and asymptotically approach unity as $k$ increases. According to the droplet theory, $\beta$ is an increasing function of the dimensionality, $\beta=(d-1) / 2$. That is, the value of 
$\beta$ ought to be small at $\sigma \lesssim 2$, but numerical data are inconsistent with the droplet theory. This inconsistency is discussed in Sec. V.

The dependence of $\beta$ on $k$ is altered between $\sigma=1.8$ and 1.7. While $\beta$ changes continuously with $k$ when $\sigma \geq 1.8, \beta$ shows a discontinuous change at a certain point when $\sigma \leq 1.7$. This intrinsic change in the $k$ dependence of $\beta$ corresponds to an outcome of the droplet theory: The density of relaxational modes switches from continuous to discrete at $d=3$ as increasing $d$. The droplet theory also declare that the relaxation is a simple exponential when $d>3$. The discrepancy between the simple exponential and stretched exponential decays in the systems at $\sigma \leq 1.7$ is discussed in Sec. $\mathrm{V}$.

\section{B. Diluted ferromagnetic model}

This subsection gives results of the 1D diluted ferromagnetic model. The system size $L$ and the parameter $\sigma$ 's are the same as in the pure ferromagnetic model. The equilibration method is also the same; the combination use of annealing and a cluster flip update is employed. The number of Monte Carlo steps is reduced to $10^{6}$ steps per sample, while the number of random samples is increased to 100 for $\sigma=2.0,1.9,1.8,1.7$ and 1.4 and 200 for $\sigma=1.6$ and 1.5, respectively.

As mentioned in Sec. IIB, we expect that the topology of the phase diagram is the same as that of the pure ferromagnetic model. Though the ordered phase is simple ferromagnetic, the dilution could change dynamical properties of the model. In fact, Huse and Fisher showed that excited large ferromagnetic droplets emerged by quenched bond disorder bring about a power-law decay [5]. The relevance of the bond dilution can be estimated by the Harris criterion [19]. Since, based on the Harris criterion, the critical exponent of the specific heat $\alpha$ of the pure ferromagnetic model is positive in $3 / 2<\sigma<2$ [10, 27, 28], autocorrelation functions are expected to show the power-law decay in the range of $\sigma$. On the other hand, we expect the exponential decay in the mean-field region $(\sigma \leq 3 / 2)$ and the KT phase $(\sigma=2)$ because the critical exponent $\alpha$ is zero in the region of $\sigma$.

Autocorrelation functions at $\sigma=2.0,1,8,1.6$, and 1.4 are plotted in Figs. 3. Using a fitting function,

$$
C(k ; t)=C_{0} \frac{\exp \left(-t / \tau_{2}(k)\right)}{\left(1+t / \tau_{1}(k)\right)^{x(k)}}+C_{\infty},
$$

I estimate characteristic times, $\tau_{1}(k)$ and $\tau_{2}(k)$, and the exponent of the power-law function, $x(k)$. The parameter $\tau_{1}(k)$ provides an indication of the waiting time for starting the power-law decay. As Figs. 3 show that autocorrelation functions of small $k$ decrease little at the beginning, and they start exhibiting power-law decay at $t \sim O\left(\tau_{1}\right)$. The introduction of the waiting time $\tau_{1}(k)$ in Eq. (25) overcomes the difficulty to fit the data which show power-law decay after a certain waiting time. The

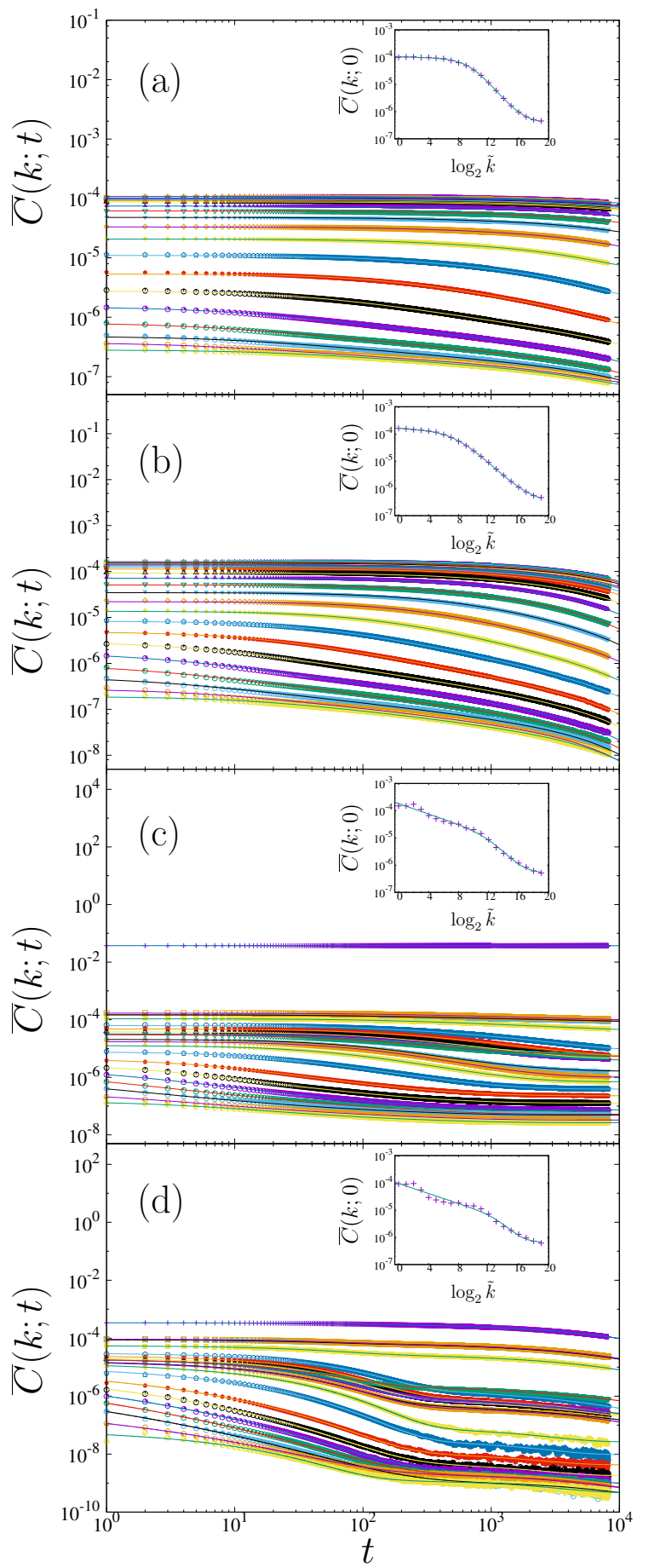

FIG. 3. (Color online) Autocorrelation functions of the diluted ferromagnetic model at (a) $\sigma=2$, (b) $\sigma=1.8$, (c) $\sigma=1.6$, and (d) $\sigma=1.4$. Insets show $k$-dependence of the autocorrelation functions at $t=0$.

parameter $\tau_{2}(k)$ is the thermal relaxation time of the autocorrelation function; the function nearly reaches thermally equilibrium value $C_{\infty}$ at $t \sim O\left(\tau_{2}\right)$. Autocorrelation functions at $\sigma \geq 3 / 2$ are well fitted by Eq. (25).

The critical exponent $\alpha$ is zero at $\sigma=2[27,28]$, so that the random dilution is irrelevant and does not change 
its universality class, Kosterlitz-Thouless phase, according to the Harris criterion [19]. But the autocorrelation functions are apparently different from the pure system at $\sigma=2$. And the data are well fitted by the powerlaw fitting form, Eq. (25). It seems inconsistent with the analytical results but it is consistent if we consider that the long-range order exists in the pure system at $\sigma=2$ due to the finite-size effect. Therefore, the finiteness of the system brings about pseudo long-range order at the marginal point $(\sigma=2)$, and the dilution gives rise to the power-law decay of pseudo long-range order.

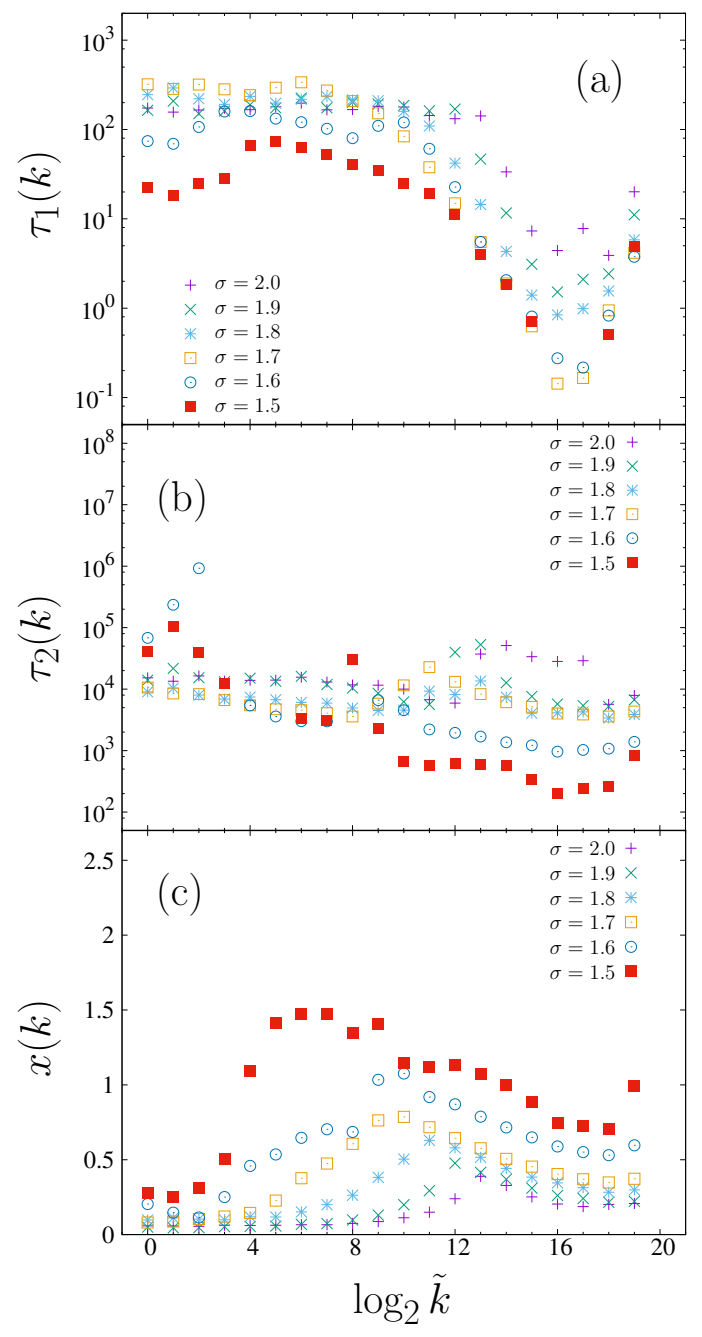

FIG. 4. (Color online) (a) Waiting times $\tau_{1}$ for several $\sigma$ 's as functions of $\tilde{k}$. At the points $\tilde{k}=16$ and 17 data of $\tau_{1}$ of $\sigma=1.5$ are not plotted since they are almost zero. (b) Lifetimes $\tau_{2}$ for several $\sigma$ 's as functions of $\tilde{k}$. (c) Exponents of the power-law decay for several $\sigma$ 's as functions of $\tilde{k}$.

The parameters $\tau_{1}(k), \tau_{2}(k)$, and the power-law exponent $x(k)$ are plotted in Figs. 4. There are autocorrelation functions that hardly decay within the prepared time window, and estimated values of $\tau_{2}(k)$ of such functions are larger than $10^{4}$. The estimated values of $\tau_{2}$ larger than $10^{4}$ are not precise, and it means that the exponential term in Eq. (25) have little contribution to the fitting analysis.

Both $\tau_{1}(k)$ and $x(k)$ are small when $\tilde{k}$ is large $(\tilde{k} \geq$ 16). The reason of small $\tau_{1}(k)$ is that small droplets in metastable state do not need long time to surmount free energy barrier and immediately start power-law relaxation to the stable states. Such small droplets frequently come out, and the autocorrelation functions of small $\tilde{k}$ count up multiple power-law decays with various onset time. This overlap of droplet relaxations causes small power-law exponent $x(k)$ at large $\tilde{k}$.

The parameter $\tau_{1}(k)$ is large when $\tilde{k}$ is small $(\tilde{k} \leq$ 4). This result is reasonable because the lifetime of metastable states of large droplets is long. Excited large droplets, therefore, do not relax readily, and we observe autocorrelations of small $k$ are almost flat in the range of $t<O\left(\tau_{1}\right)$. The multiple parameter fitting with Eq. (25) erroneously infers that the exponent $x(k)$ is small when $\tilde{k}$ is small. The reason of the improper inference is that the autocorrelation functions hardly exhibit power-law decay within the time window, and the exponent is estimated to be smaller than real value. In fact, the autocorrelation functions of small $\tilde{k}$ exhibit power-law decay with $x(k) \sim 1$ when the system size is small enough [29].

A marked feature of the autocorrelation functions of the diluted system is that they are placed with roughly equal intervals. Since scaled wave numbers $\tilde{k}$ are chosen from the power-of-two, the functions are approximately described by

$$
C(\tilde{k} ; t) \sim \tilde{k}^{-y} C(\tilde{k}=1 ; t),
$$

where $y$ is a constant. The fact that the autocorrelation function is roughly proportional to the power of $\tilde{k}$ means excited droplets have a fractal-like structure. The autocorrelation functions at $t=0$ are plotted the insets of Figs. 3 as functions of $\tilde{k}$. It can be seem from the insets that the profiles are quite similar to that of the pure system at $\sigma=2$ which is located in KT phase. However, the origin of the KT phase like feature in diluted systems is different from that of the pure system. Details of the origin will be discussed in Sec. V.

The autocorrelation functions at $\sigma=1.4$ (Figs. 3(d)) do not exhibit power-law decay but the functions are well described by the sum of two stretched exponential functions [Eq. (24)]. The change from the power-law to the stretched exponential decay is expected since randomly placed diluted interactions are averaged out in the meanfield limit. Contrary to the relaxation process, the autocorrelation functions still have a fractal-like structure. It means that the shape of excited droplets still keeps a fractal-like structure though their dynamics are altered.

\section{Spin glass model}

The results of the spin glass model is presented in this subsection. The system size and Monte Carlo steps are 
significantly reduced since the decay of the long-range interactions is slow (the range of the interaction parameter is $\sigma \leq 1)$ comparing to the pure and diluted ferromagnetic models. The system size is set as $L=2^{13}(=8192)$. During the equilibration, spins are updated by the singlespin-flip algorithm because the simple cluster flip update does not work in frustrated spin systems. To accelerate relaxation the replica exchange Monte Carlo method is used [30]. For the measurement of autocorrelation functions, $10^{6}$ Monte Carlo steps are executed. The number of random samples is 100 .

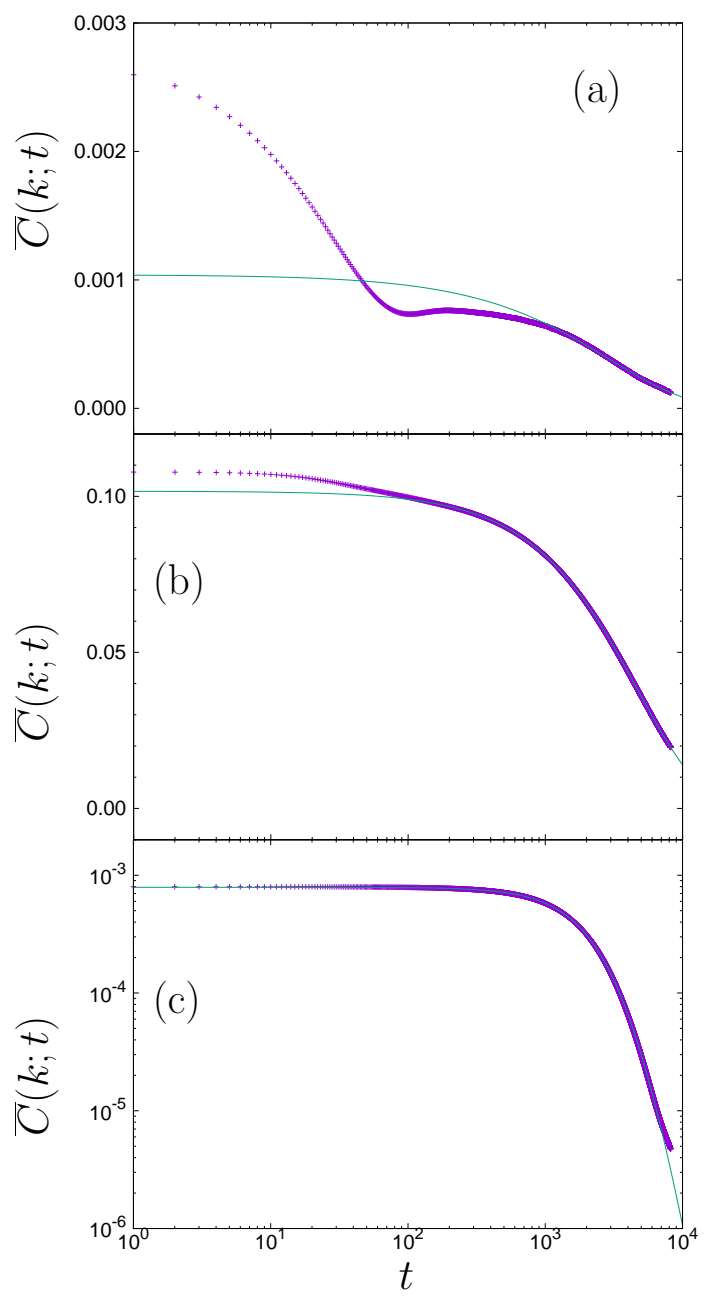

FIG. 5. (Color online) Autocorrelation functions of the spin glass model at (a) $\sigma=0.9$, (b) $\sigma=0.7$, and (c) $\sigma=0.6$. Stretching exponential curves in (a) and (b) are fitting results of the $\alpha$-relaxation regime. Curve in (c) is obtained by using the Havriliak-Negami type fitting function.

At the lower critical value of $\sigma(=1)$, Moore showed that there is no spin glass phase at a finite temperature [31]. However we observe a pseudo spin glass transition at a finite temperature which seems to be independent from the system size since the correlation length diverges extremely rapidly as lowering the temperature. Figures 5 show the autocorrelation functions at $\sigma=0.9,0.7$, and
0.6. Autocorrelation functions of $k>0$ are omitted since they are fairly small comparing to that of $k=0$. The autocorrelation function at $\sigma=0.9$ shows typical spinglass behaviors [32]: It shows a ballistic relaxation at the beginning, and go into a plateau regime, $\beta$-relaxation regime, through a small dip, boson peak. At the end, it shows a slow relaxation, $\alpha$-relaxation regime, which is well described by a stretched exponential form [Eq. (23)] Curves in Figs. [5(a) and (b) show fitting results for $\alpha$ relaxation. The boson peak disappears when $\sigma \leq 0.8$, and the autocorrelation decays right after the ballistic relaxation. Though the microscopic origin of the boson peak has been a debating issue, it relates to relaxations in locally restricted area. At $\sigma=0.6$, the mean-field region, the correlation hardly decays at the beginning, and suddenly start decaying around $t \sim 1000$. While the autocorrelation function cannot be fitted by exponential and power functions, the Havriliak-Negami type 33] fitting function,

$$
C(t)=\frac{C_{0}}{\left[1+(t / \tau)^{\gamma}\right]^{x}}
$$

well fits the data. The resulting parameters are $\tau=$ $4.58 \times 10^{3}, \gamma=1.69$, and $x=4.24$, respectively. The autocorrelation functions of the spin glass model are different from those suggested by the droplet theory [see Sec. IIC. Reasons are considered in the next section.

\section{DISCUSSION}

In this section, we first examine reasons of discrepancies between the droplet theory and numerical data of pure ferromagnetic model shown in Sec. IVA. In Sec. IVB, autocorrelation functions of the diluted ferromagnetic model exhibit KT-like features, the powerlaw decay and a power-law form of the structure factor. Considering the origin of KT-like features, though the ordered phase of the system seems KT phase, it is revealed that the origin of the KT-like features is a crossover effect caused by randomly diluted lattice. The discrepancy between droplet theory and numerical data in the spin glass model is discussed in the last part of this section.

First we consider the reason why two exponential functions are required when we attempted to figure out what the function form describes well numerical data of the pure ferromagnetic model in $\sigma \leq 1.6$. The function form in the region does not conform a result from the droplet theory, that the autocorrelation function is described by the simple exponential function when $d \geq 3$. This threshold dimension is lower than the upper critical dimension, $d_{u}=4$, and the corresponding value of $\sigma$, therefore, would be slightly larger than $\sigma_{u}=3 / 2$. The value of $\sigma$ at the threshold, $\sigma_{c}$, is presumably close to 1.6 , and it is probable that the change of the function form relates to the intrinsic change of the function form at the threshold. 


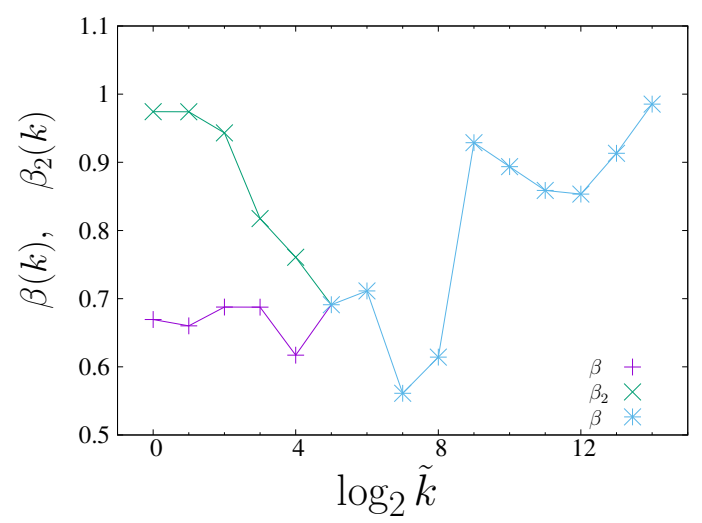

FIG. 6. (Color online) Plot of stretching exponents $\beta$ and $\beta_{2}$ at $\sigma=1.6$. While two stretching exponential functions are needed to fit data in $\tilde{k} \leq 4$ [Eq. (24)], preparing a stretching exponential function is sufficient to fit data in $\tilde{k} \geq 5$.

The function form is the simple exponential function in the mean-field model because the model reduces a manybody dynamics to a one-body dynamics. Therefore, it is naively expected that autocorrelation functions are simple exponential when $\sigma \leq \sigma_{u}$. However, autocorrelation functions at $\sigma=1.4$ and 1.5 (not shown) are essentially the same as those at $\sigma=1.6$. Figure 6 shows $\beta(\tilde{k})$ and $\beta_{2}(\tilde{k})$ at $\sigma=1.6$. $\beta_{2}$ is nearly unity when $\tilde{k}$ is small, it decreases as increasing $\tilde{k}$, and it merges to $\beta$ at $\tilde{k}=5$. The stretched exponential function with $\beta$ seems an extra relaxation function if we assume the stretched exponential function with $\beta_{2}$ is derived from the simple exponential function predicted by the droplet theory. The extra unexpected relaxation would come from the ununiformity in interactions which form surface of droplet. While the droplet theory supposes the surface dimension of droplets is $d-1$, the surface dimension of droplets in our 1D model is not obvious; a plausible definition is a value of integral of interactions which extend outward from a droplet,

$$
I=\int_{i \in \mathcal{C} \wedge j \notin \mathcal{C}} \frac{J}{r_{i j}^{\sigma}} d r_{i j}
$$

where $i$ runs from one end of the droplet to the other end, and $j$ runs all over the sites except on the droplet. If the droplet consists of a sequentially aligned Ising spins, the integral value is proportional to $c^{2-\sigma}$, where $c$ is a volume of the droplet. Assuming the volume is proportional to the power of $d$, the surface dimension of the droplet is estimated as $d(2-\sigma)$. The result seems reasonable at both extremities of $\sigma$; the integral value is equal to a constant at $\sigma=2$ and is proportional to $d$ at $\sigma=1$. However, the value is unexpectedly small at the marginal boundary of the mean-field region, $\sigma=3 / 2$. At the boundary the effective dimension of the volume of droplets is four, so that we obtain $d(2-\sigma)=2$ as the surface dimension. If the surface dimension is strictly $d(2-\sigma)$, the dynamics should change at $\sigma=3 / 2$; the system exhibits the stretched exponential decay in $\sigma>3 / 2$, the simple exponential decay in $\sigma<3 / 2$, respectively. However, numerical data are inconsistent with the result. The inconsistency is probably caused by the fluctuation of the surface dimension of droplets. The surface dimension, $d(2-\sigma)$, is estimated by assuming that droplets consist of sequentially aligned Ising spins. Actually most of droplets consist of a lot of fractions of sequential Ising spin sites, so that the surface dimension of these droplets are larger than the estimated value. Additionally, relatively small fractions of Ising spin sites contribute to lowering the effective surface dimension because their surfaces are softer and fluctuate widely. As a result, effective surface dimension of droplets has a distribution even though the value of $\sigma$ is fixed. The distribution brings about two relaxation forms, the simple and the stretched exponential forms near the boundary $(\sigma=3 / 2)$.

Next we consider reasons that the disagreement in the stretching exponent $\beta$ between numerical data and the droplet theory at $\sigma \lesssim 2$. While the droplet theory gives $\beta=(d-1) / 2(\gtrsim 0$ at $\sigma \lesssim 2)$, numerically obtained $\beta$ 's are fairly larger than the expected value. An apparent disagreement in numerical data is that the relaxation at the beginning is considerably slow. According to the droplet theory, the logarithmic derivative of the function is

$$
\frac{d}{d t} \ln C(k ; t) \propto-\beta\left(\frac{t}{\tau}\right)^{\beta-1}
$$

Therefore, the slope of the autocorrelation function should be steep at the beginning $(t \ll \tau)$ if $\beta$ is nearly zero as given by the droplet theory. The absence of the steep decay is caused by the discreteness of the Ising variable. As shown in the inset of Fig. 1(a), the emergence of small sized droplets are suppressed, though the phase is supposed to be in the KT phase at $\sigma=2$. While the continuous spin variable as in the XY model is able to contribute to the steep decay by creation and annihilation of spin waves, these small energy contributions are absent in the Ising spin system. The effect of the discreteness is reduced when the temperature is close enough to the critical point; that is, thermal fluctuations blur the discreteness of Ising spin, so that the structure factor is expressed by the power of the wave length. In such a nearly critical system, the steep decay, which coincides with a power-law decay at the criticality, should be observed since large $k$ droplet excitations decay at the beginning. Since the system at $\sigma 1.9$ has the true long-range order, the tail of the structure factor is not long as that of the KT phase. However, a small stretching exponent would be observed if the temperature of the ordered phase is high enough to blur the discreteness of the Ising spin.

The autocorrelation functions of the diluted model show the power-law decay and the fractal-like structure though the system is in the ferromagnetic ordered state. It is known that these pseudo critical features are caused by randomly diluted lattice [34]. When the degree of dilution is slightly lower than the critical point, the correlation length between lattice sites is given by

$$
\xi_{p} \sim\left(p-p_{c}\right)^{-\nu_{p}},
$$


where $p$ is the concentration of lattice sites, $p_{c}$ is the critical concentration of lattice sites, and $\nu_{p}$ is the critical exponent of the percolation correlation length. The ordered region is proportional to the number of lattice sites, when ferromagnetic regions are larger than $\xi_{p}$. On the other hand, the ordered region forms a fractal structure, when ferromagnetic regions are smaller than $\xi_{p}$. Thus, while the structure factor shows the Lorentzian peak shape in $k<1 / \xi_{p}$, it shows the power-law shape in $k>1 / \xi_{p}$. The structure factor of the diluted model seems to be classified into three regions. As an example, the structure factor at $\sigma=1.6$ in the inset of Fig. 3)(c) is classified as follows: (i) it is suppressed by the discreteness of Ising spin in $\tilde{k} \geq 12$, (ii) it is almost a constant in $\tilde{k} \leq 2$, and (iii) it shows the power-law shape in $2<\tilde{k}<11$. This classification indicates the percolation correlation length is estimated as roughly $L / 8$ when $\sigma=1.6$. The relaxation also depends on $k$; relaxations of autocorrelations in $\tilde{k} \leq 2$ are considerably slower than those in $\tilde{k}>2$ [see Fig. 3)(c) and Fig. 44(c)]. Essentially the same behaviors are also observed at $\sigma=1.4$. Though effects of the random dilution is averaged out in the mean-field limit, as discussed above, fractal-like features still remain at $\sigma=1.4$ due to the distribution of the surface dimension of droplet. Whereas slow decay in autocorrelation functions of $\tilde{k}<1 / \xi_{p}$ is observed at $\sigma \leq 1.6$, there is no such a signal at $\sigma>1.6$. The difference is caused by that $\xi_{p}$ becomes larger as increasing $\sigma$. The transition temperature decreases as increasing $\sigma$, and thermal fluctuations become weaker. The system comes close to the percolation transition point as decreasing the transition temperature, and the approaching to the point causes the growth of $\xi_{p}$. As a result, the autocorrelation functions at $\sigma>1.6$ seems like fractal in all the region of $\tilde{k}$.

The fractal-like features are also observed in disordered ferroelectrics. Koreeda and collaborators observed the power-law distribution in the quasielastic light scattering in $\mathrm{Pb}\left(\mathrm{Mn}_{1 / 3} \mathrm{Nb}_{2 / 3}\right) \mathrm{O}_{3}$, and they also observed the power exponent of the spectrum depends on temperature [35]. Their observations are indeed the same as those of results that the droplet theory gives. Dynamics of disordered ferroelectrics are not understood well. To study of the dynamics, spin glass models are often employed since they exhibit spin glass like behaviors, extremely slow dynamics which involve aging, memory effect, frequency dependence of the AC susceptibility, et al. However, considering the experimental results by Koreeda and collaborators and the results from diluted ferromagnetic models, it should be examined which models is proper to describe phenomena of interest.

Autocorrelation functions of the spin glass model obtained by the numerical simulation are quite different from those given by the droplet theory. While their decay form is given by the power of logarithmic function of time according to the droplet theory, numerical data exhibit the stretched exponential decay in the range of $0.7 \leq \sigma \leq 1$ and Havriliak-Negami type relaxation at $\sigma=0.6[$ Eq. (27)] . The disagreement would come from an intrinsic difference between microscopic and macroscopic variables, which makes little difference in ferromagnetic phase. While, in this paper, the EdwardsAnderson (EA) order parameter [25] [Eq. (20)] is employed as a macroscopic variable to investigate dynamical properties, the droplet theory gives the time-delayed correlation of a local spin variable. The EA order parameter measures a similarity between replicated systems which have the same set of interactions $\left\{J_{i j}\right\}$. Therefore, the autocorrelation of the EA order parameter measures the time-delayed correlation of the similarity. On the other hand, the time-delayed correlation of a local spin variable measures a local spin dynamics in a sample. Thus, autocorrelations of the two observables exhibit different relaxations.

\section{SUMMARY}

In this paper, Monte Carlo simulations on 1D Ising models with long-range interactions are executed, and the numerical data are compared with results given by the droplet theory. Essentially consistent results are obtained in the pure and the diluted ferromagnetic models, though disagreements caused by the discreteness of Ising spin and the distribution of the surface dimensionality are also observed. Numerical results in the spin glass model are different from results given by the droplet theory. This means that dynamics of the macroscopic variable are essentially different from those of the microscopic variable analyzed in the droplet theory.

It is shown that combined use of the droplet theory and numerical simulation helps us to understand nature of ordered states. Applying the results to investigations of non-trivial phases as in disordered ferroelectrics [35] or frustrated magnets [36] could advance understanding of ordered states. To extend the range of applicable fields, clarifying relations between ordered states and its dynamics in other fundamental spin models is required.

\section{ACKNOWLEDGMENTS}

The author thanks Prof. Takayama and Dr. Nonomura for useful comments on the manuscript. The random-number generator MTGP23209 [37] was used for numerical calculations.

\section{Appendix: $O(N)$ Monte Carlo method}

The main idea of the $O(N)$ MC method is to estimate a molecular field at site $i$ by sampling interacting sites. The Hamiltonian of the Ising model with long-range interactions is given by

$$
\mathcal{H}=-\sum_{i<j} J_{i j} S_{i} S_{j}
$$


The summation in Eq. A.1 runs over every pair of interactions: therefore, interactions $J_{i j}$ should not be those of bare but of rescaled when the periodic boundary condition is imposed. We suppose all the interactions are ferromagnetic $\left(J_{i j} \geq 0\right)$ for convenience in explaining. The extension to spin glass model is given at the bottom of Appendix.
The Boltzmann weight $W_{\mathrm{B}}(\{S\})$ for site $i$ is given by

$$
\begin{aligned}
W_{\mathrm{B}}(\{S\}) & =\prod_{j(\neq i)} \exp \left(\beta J_{i j} S_{i} S_{j}\right) \\
& =\prod_{j(\neq i)}\left[\frac{1+S_{i} S_{j}}{2} e^{\beta J_{i j}}+\frac{1-S_{i} S_{j}}{2} e^{-\beta J_{i j}}\right],
\end{aligned}
$$

where $\{S\}$ represents a spin configuration. By introducing an auxiliary parameter $\alpha(>0)$, we are able to deactivate interactions stochastically, and the deactivation reduces the computational cost with the stochastic legitimacy. After introducing the parameter $\alpha$, the resulting $W_{\mathrm{B}}(\{S\})$ is written by [10]

$$
W_{\mathrm{B}}(\{S\}) \propto \sum_{\{k\}} P\left(\lambda_{\text {tot }} ; k_{\text {tot }}\right) f(\{k\} ;\{\lambda\}) w(\{k\} ;\{\lambda\} ;\{S\}) .
$$

Here, $P\left(\lambda_{\text {tot }} ; k_{\text {tot }}\right), f(\{k\} ;\{\lambda\})$, and $w(\{k\} ;\{\lambda\} ;\{S\})$ are the Poisson probability mass function, the multinomial probability mass function, and a weight function, respectively. The explicit forms of functions are as follows:

$$
\begin{aligned}
& P\left(\lambda_{\text {tot }} ; k_{\text {tot }}\right)=e^{-\lambda_{\text {tot }}} \frac{\lambda_{\text {tot }}^{k_{\text {tot }}}}{k_{\text {tot }} !} \\
& f(\{k\} ;\{\lambda\})=k_{\text {tot }} ! \prod_{j(\neq i)}\left[\frac{1}{k_{i j} !}\left(\frac{\lambda_{i j}}{\lambda_{\text {tot }}}\right)^{k_{i j}}\right], \\
& w(\{k\} ;\{\lambda\} ;\{S\})=\prod_{j(\neq i)}\left[\frac{1+S_{i} S_{j}}{2}+\frac{1-S_{i} S_{j}}{2}\left(\frac{\alpha}{\lambda_{i j}}\right)^{k_{i j}}\right],
\end{aligned}
$$

where $\lambda_{i j}=2 \beta J_{i j}+\alpha, \lambda_{\text {tot }}=\sum_{j(\neq i)} \lambda_{i j}$, and $k_{\text {tot }}=$ $\sum_{j(\neq i)} k_{i j}$. The variable $k_{i j}$ is a number of activated bonds between $i$ and $j$, and a set $\{k\}$ represents an activated bond configuration.

$P\left(\lambda_{\text {tot }} ; k_{\text {tot }}\right)$ gives a stochastic weight of a number of total bonds, $k_{\text {tot }}$, whose mean value is $\lambda_{\text {tot }}$. The constant $\lambda_{\text {tot }}$ is the summation of an effective interaction $\lambda_{i j}$. In the $O(N)$ MC method, an effective interaction $\lambda_{i j}$ appears as a summation of the bare interaction $\beta J_{i j}$ and an auxiliary tunable interaction $\alpha$. The auxiliary parameter $\alpha$ determines the efficiency of the $O(N) \mathrm{MC}$ method. Decreasing the value of $\alpha$ decreases the number of active bonds, and it reduces the computational cost relating to the bond activation. However, small $\alpha$ brings about a low acceptance ratio for a spin flip. Indeed, in the limit of $\alpha=0$, the $O(N)$ MC method corresponds to the Swendsen-Wang cluster MC method [38]. Conversely, increasing the value of $\alpha$ raises an acceptance ratio for a spin flip, but it also raises the number of active bonds and the computational cost. In fact, the limit of $\alpha=\infty$ reduces the $O(N)$ method to the Metropolis method. The dynamics of the simulation is optimized by tuning the parameter $\alpha$. A reasonable choice of $\alpha$ is

$$
\alpha_{i j}=2 \beta J_{i j} \tilde{\alpha}
$$

where $\tilde{\alpha}$ is a constant. This choice makes $\lambda_{i j}$ proportional to $\beta J_{i j}$ as

$$
\lambda_{i j}=2 \beta J_{i j}(1+\tilde{\alpha})
$$

this means that all of the bonds are activated with probabilities proportional to the bare interaction $\beta J_{i j}$. Using the choice, functions $f(\{k\} ;\{\lambda\})$ and $w(\{k\} ;\{\lambda\} ;\{S\})$ 
are rewritten, respectively, by

$$
\begin{aligned}
f(\{k\} ;\{\lambda\}) & =k_{\text {tot }} ! \prod_{j(\neq i)}\left[\frac{1}{k_{i j} !}\left(\frac{J_{i j}}{J_{\mathrm{tot}}}\right)^{k_{i j}}\right], \\
w(\{k\} ;\{\lambda\} ;\{S\}) & =\prod_{j(\neq i)}\left[\frac{1+S_{i} S_{j}}{2}+\frac{1-S_{i} S_{j}}{2} \kappa^{k_{i j}}\right] \\
& =\prod_{j \in\left(S_{j+1} S_{i}\right)} \kappa^{k_{i j}}
\end{aligned}
$$

where $J_{\text {tot }}=\sum_{j(\neq i)} J_{i j}$ and $\kappa=\tilde{\alpha} /(1+\tilde{\alpha})$, and the product in Eq. A.10 runs over antiparallel spin pairs. Equation A.9 gives a stochastic distribution so that $k_{i j} \propto J_{i j}$. A stochastic weight of a spin configuration $\{S\}$ in a bond configuration $\{k\}$ is given by Eq. (A.10).

Using the Eqs. (A.4), A.9 and A.10, a pseudocode of a heat-bath spin update with the $O(N)$ MC method is implemented as follows:

$$
\begin{aligned}
& p:=1 \\
& q:=1 \\
& c_{p}^{\prime \prime}:=2 \tilde{\alpha}+2 \\
& c_{q}^{\prime \prime}:=2 \tilde{\alpha} \\
& c_{p}^{H}:=2 \tilde{\alpha}^{2}+2 \tilde{\alpha}+1 \\
& c_{q}^{H}:=2 \tilde{\alpha}^{2}+4 \tilde{\alpha}+1 \\
& k_{\text {tot }}:=\operatorname{Poisson}\left(\lambda_{\text {tot }}\right) \\
& \text { for } k=1 \text { to } k_{\text {tot do }} \\
& j:=\text { Walker }(\{\lambda\}) \\
& \text { if } S_{j}=S_{i} \text { then } \\
& p:=c_{p}^{\prime \prime} p \\
& q:=c_{q}^{\prime \prime} q \\
& \text { else } \\
& p:=c_{p}^{H} p \\
& q:=c_{q}^{H} q \\
& \text { end if } \\
& \text { end for } \\
& \text { if Random }()>\frac{p}{p+q} \text { then } S_{i}:=-S_{i}
\end{aligned}
$$

A formula $a:=n$ means that $n$ is plugged in for $a$. Poisson $\left(\lambda_{\text {tot }}\right)$ generates a random number from a Pois- son distribution with a mean of $\lambda_{\text {tot }}$, Walker $(\{\lambda\})$ generates a random number from a distribution given by Eq. (A.9) 39]. Random() generates a random number from a uniform distribution in the range from 0 to 1.

Since the cost of $O(N)$ MC method for interaction pairs which give $\lambda_{i j}>1$ is more expensive than that of the conventional MC method, switching the stochastic weight from the one of the $O(N)$ MC method to the conventional one reduces the cost of computation. For the combination use, we introduce an arbitrary constant $c$ and separate interacting pairs into $\{i j\}_{<c}$, a set of pairs giving $\lambda_{i j}<c$, and $\{i j\}_{\geq c}$, the set of remaining pairs giving $\lambda_{i j} \geq c$. Then, the spin flip probability, $p /(p+q)$, is replaced by $p /(p+q \exp \{-\beta \Delta E\})$, where $\Delta E$ is the single-spin-flip energy difference for $\{i j\} \geq c$.

When an interaction $J_{i j}$ is randomly diluted to $\gamma J_{i j}(0 \leq \gamma<1)$, the multinomial probability mass function, $f(\{k\} ;\{\lambda\})$, becomes dependent on the site $i$. If we modify the function for each site $i$, a large memory area which is proportional to $O\left(N^{2}\right)$ is needed. We can save the large memory area by changing the multiplicative factors, $c$ 's in the pseudocode, as

$$
\begin{aligned}
c_{p}^{\prime \prime} & :=2 \tilde{\alpha}+1+\gamma, \\
c_{q}^{\prime \prime} & :=2 \tilde{\alpha}+1-\gamma, \\
c_{p}^{\sharp} & :=2 \tilde{\alpha}^{2}+(3-\gamma) \tilde{\alpha}+1, \\
c_{q}^{\sharp} & :=2 \tilde{\alpha}^{2}+(3+\gamma) \tilde{\alpha}+1 .
\end{aligned}
$$

The constant $\gamma$ substitutes the change in $f(\{k\} ;\{\lambda\})$ with the spin-flip probability. Employing the modification of the multiplicative factors, we can use the same $f(\{k\} ;\{\lambda\})$ as the pure system for the diluted spin system.

For the antiferromagnetic system, the satisfied spin configuration inverts from parallel to antiparallel, so that the weight function for the system is converted to

$$
w(\{k\} ;\{\lambda\} ;\{S\})=\prod_{j(\neq i)}\left[\frac{1+S_{i} S_{j}}{2} \kappa^{k_{i j}}+\frac{1-S_{i} S_{j}}{2}\right] .
$$

For the spin glass model, we apply Eq. A.10 and Eq. A.11 for ferromagnetic and antiferromagnetic interactions, respectively.
[1] K. S. Cole and R. H. Cole, J. Chem. Phys. 9, 341 (1941).

[2] C. A. Angell, K. L. Ngai, G. B. McKenna, P. F. McMillan, and S. W. Martin, J. Appl. Phys. 88, 3113 (2000).

[3] P. C. Hohenberg and B. I. Halperin, Rev. Mod. Phys. 49, 435 (1977).

[4] Y. Ozeki and N. Ito, J. Phys. A: Math. Theor. 40, R149 (2007).

[5] D. A. Huse and D. S. Fisher, Phys. Rev. B 35, 6841 (1987).

[6] C. Tang, H. Nakanishi, and J. S. Langer, Phys. Rev. A 40, 995 (1989).
[7] D. S. Fisher and D. A. Huse, Phys. Rev. Lett. 56, 1601 (1986).

[8] D. S. Fisher and D. A. Huse, Phys. Rev. B 38, 386 (1988).

[9] K. Fukui and S. Todo, J. Comput. Phys. 228, 2629 (2009).

[10] Y. Tomita, J. Phys. Soc. Jpn. 78, 014002 (2009).

[11] Y. Tomita, J. Phys. Soc. Jpn. 78, 114004 (2009).

[12] D. J. Thouless, Phys. Rev. 187, 732 (1969).

[13] P. W. Anderson and G. Yuval, J. Phys. C 4, 607 (1971).

[14] J. M. Kosterlitz, Phys. Rev. Lett. 37, 1577 (1976).

[15] R. J. Glauber, J. Math. Phys. 4, 294 (1963). 
[16] M. Suzuki and R. Kubo, J. Phys. Soc. Jpn. 24, 51 (1968).

[17] K. Kawasaki and T. Yamada, Prog. Theor. Phys. 39, 1 (1968).

[18] R. Abe, Prog. Theor. Phys. 39, 947 (1968).

[19] A. B. Harris, J. Phys. C 7, 1671 (1974).

[20] B. Berche, A. I. Fariñas-Sánchez, Y. Holovatch, and R. Paredes V., Eur. Phys. J. B 36, 91 (2003).

[21] T. Surungan and Y. Okabe, Phys. Rev. B 71, 184438 (2005).

[22] G. Kotliar, P. W. Anderson, and D. L. Stein, Phys. Rev. B 27, 602 (1983).

[23] S. Kirkpatrick and D. Sherrington, Phys. Rev. B 17, 4384 (1978).

[24] H. G. Katzgraber and A. P. Young, Phys. Rev. B 67, 134410 (2003).

[25] S. F. Edwards and P. W. Anderson, J. Phys. F 5, 965 (1975).

[26] W. Gebhardt and U. Krey, Phasenübergänge und kritische Phänomene. Eine Einführung für Physiker im Hauptstudium. (Friedrich Vieweg \& Sohn, Braunschweig/Wiesbaden, 1980).

[27] M. E. Fisher, S. Ma, and B. G. Nickel, Phys. Rev. Lett.
29, 917 (1972).

[28] M. Suzuki, Prog. Theor. Phys. 49, 424 (1973).

[29] To be published in Ferroelectrics.

[30] K. Hukushima and K. Nemoto, J. Phys. Soc. Jpn. 65, 1604 (1996).

[31] M. A. Moore, Phys. Rev. B 82, 014417 (2010).

[32] K. Binder and W. Kob, Glassy Materials and Disordered Solids, 2nd ed. (World Scientific, Singapore, 2011).

[33] S. Havriliak and S. Negami, Polymer 8, 161 (1967).

[34] H. Ikeda, K. Iwasa, and K. H. Andersen, J. Phys. Soc. Jpn. 62, 3832 (1993).

[35] A. Koreeda, H. Taniguchi, S. Saikan, and M. Itoh, Phys. Rev. Lett. 109, 197601 (2012).

[36] H. Shinaoka, Y. Tomita, and Y. Motome, Phys. Rev. B 90, 165119 (2014).

[37] M. Saito and M. Matsumoto, ACM Trans. Math. Softw. 39, 12 (2013).

[38] R. H. Swendsen and J. S. Wang, Phys. Rev. Lett. 58, 86 (1987).

[39] A. J. Walker, ACM Trans. Math. Software 3, 253 (1977). 